\title{
Multiple synchronization attractors of serially connected spin-torque nanooscillators
}

\author{
Dong Li, ${ }^{1}$ Yan Zhou, ${ }^{2}$ Bambi Hu, ${ }^{3}$ Johan Åkerman, ${ }^{4}$ and Changsong Zhou ${ }^{1, *}$ \\ ${ }^{1}$ Department of Physics, Centre for Nonlinear Studies and The Beijing-Hong Kong-Singapore Joint Centre for Nonlinear and Complex \\ System (Hong Kong), Hong Kong Baptist University, Kowloon Tong, Hong Kong \\ ${ }^{2}$ Department of Physics, The University of Hong Kong, Pokfulam Road, Hong Kong \\ ${ }^{3}$ Department of Physics, University of Houston, Houston, Texas 77204-5005, USA \\ ${ }^{4}$ Department of Physics, University of Gothenburg, SE-412 96 Gothenburg, Sweden
}

(Received 10 November 2011; revised manuscript received 11 June 2012; published 18 July 2012)

\begin{abstract}
Spin-torque nanooscillators (STNOs), which have both the common properties of nanosized oscillators (small size, tunable operating frequency) and some particular ones (wide operating range, easy on-chip integration, etc.), have received a great deal of attention due to their high potential in applications. Yet synchronization of serially connected STNOs has been considered essential for applications. In this paper, we present findings concerning the following properties of synchronized serially connected STNOs: (i) multiple synchronization attractors coexist, and the attracting basins are entangled in a complicated manner; (ii) these attractors have different synchronized frequencies and output powers; and (iii) switching among these attractors can be induced by a small noise, which causes a resonance peak in the power spectra to vanish. These characteristics can be understood using saddle-node bifurcations and have direct impact on laboratory experiments and the potential applications of STNO-based devices.
\end{abstract}

DOI: 10.1103/PhysRevB.86.014418

PACS number(s): 85.75.Bb, 05.45.Xt, 75.40.Gb, 62.25.-g

\section{INTRODUCTION}

Various types of nanosized oscillators are currently attracting great interest. They lend themselves to many possible applications, thanks to their common properties, which include small size, tunable operating frequency, etc. ${ }^{1-12}$ Spin-torque nanooscillators (STNOs), ${ }^{1-6}$ which are nanosized spintronic devices capable of microwave generation at frequencies in the gigahertz-terahertz range with high quality factors, show great potential for wireless and radar communication. The collective behavior of an array of coupled STNOs is also significant for many applications: For example, its magnetic oscillation can be used as an information carrier in spin-based logic circuits ${ }^{13-17}$ and polychronous wave computation, ${ }^{18}$ as well as serve as a model system for fundamental study. ${ }^{19}$ More importantly, synchronization of coupled STNO arrays has been demonstrated as a technique essential for potential microwave generation applications, as it leads to increased power and improved signal purity and quality, ${ }^{20-23}$ in contrast with the very limited output power and relatively large linewidth of a single STNO. ${ }^{19,24-26}$ However, despite much experimental work in this direction, the dynamical characteristics of the synchronization states are still unclear. It is therefore imperative to gain insight into the synchronization attractors of coupled STNO networks in order to design and optimize STNO-based novel spintronic radio-frequency devices for next-generation microwave applications.

In this paper, we study the dynamical behaviors which arise in the synchronization region of serially connected STNO networks, where all the STNOs oscillate at a uniform synchronized frequency. It is found that multiple attractors with different phase shifts, synchronized frequencies, and resonance peak amplitudes can coexist in such a system across quite a broad range of system parameters. Both in-phase and antiphase synchronization can be observed in pairs of serially connected STNOs. The attracting basins are entangled in a complicated manner. A random switching between these two states can be induced by means of a small noise, which causes the resonance peak in the power spectra to vanish. When the number of STNOs is greater than two, the attractors become more complex. In contrast to the previously used Hopf bifurcation theory of coupled phase oscillators, ${ }^{27,28}$ we show that these results can be precisely understood by a model based on saddle-node bifurcation theory.

Our results are significant for the improved understanding of the nonlinear characteristics of such STNO devices, and have a direct impact on experiments and applications involving STNO systems. We show that the synchronization of serially connected STNOs does not necessary increase the resonance peak, which was one of the original motivations for synchronizing STNOs. One of the common methods of identifying synchronization in such coupled nanosized high-frequency oscillator systems is by measuring the resonance peak in experimental power spectra, yet this becomes impractical when the peak vanishes. In addition, these findings may have some potential in STNO-based information processing and digital computation applications.

\section{STNO MODEL}

In this work, we investigate an in-plane STNO model, as shown in Fig. 1(a). Since we focus on the interaction properties of electrically connected STNOs, the detailed micromagnetics in each free layer is neglected and we adopt a macrospin model to capture the main physics governing the free layer magnetodynamics, where the unit vector of the free layer magnetization $\mathbf{m}$ is described by the Landau-LifshitzGilbert-Slonczewski equation, ${ }^{29}$

$$
\frac{d \mathbf{m}}{d t}=-|\gamma| \mathbf{m} \times \mathbf{H}_{\mathrm{eff}}+\alpha \mathbf{m} \times \frac{d \mathbf{m}}{d t}+|\gamma| \beta J \mathbf{m} \times(\mathbf{m} \times \mathbf{M}),
$$

where $\gamma$ is the gyromagnetic ratio, $\alpha$ is the Gilbert damping parameter, and $\beta$ contains material parameters and 

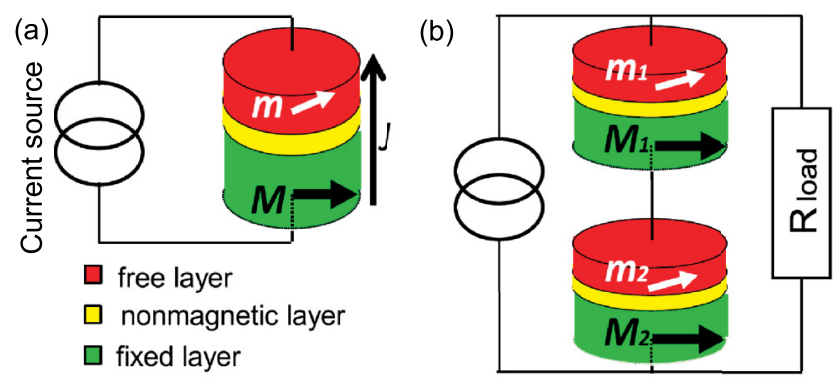

FIG. 1. (Color online) (a) Schematic of an in-plane STNO. The free layer magnetization $\mathbf{m}$ is separated from the fixed layer $\mathbf{M}$ by a nonmagnetic layer. (b) Two serially connected STNOs.

fundamental constants. ${ }^{20}$ The electrical current $J$ is defined as positive when electrons flow from the fixed layer to the free layer. The effective field $\mathbf{H}_{\text {eff }}$ carries the contribution of an external applied magnetic field $H_{a}$, an anisotropy (easy axis) field $H_{k}$ along the $x$ axis, and a demagnetization (easy plane anisotropy) field $H_{d z} \approx 4 \pi M_{s}$, where $M_{s}$ is the saturation magnetization of the free layer material. We thus get $\mathbf{H}_{\mathrm{eff}}=H_{a} \hat{e}_{x}+\left(H_{k} m_{x} \hat{e}_{x}-H_{d z} m_{z} \hat{e}_{z}\right) /|\mathbf{m}|$. In the case of a serial circuit [shown in Fig. 1(b)], the shared current in the STNO branch is ${ }^{20,21}$

$$
J(t)=J_{d} /\left[C_{a}-C_{b} \sum_{i} \cos \psi_{i}(t)\right],
$$

where $\psi_{i}$ is the angle from $\mathbf{M}$ to $\mathbf{m}$ of the $i$ th STNO, and $C_{a}$ and $C_{b}$ are constants. Such a macrospin model is valid when the spatial distribution of the magnetizations in the free layer is neglected and it has been successfully employed in interpreting a wide range of magnetic and more recent spin-torque related experiments. ${ }^{20,30,31}$

\section{SYNCHRONIZATION ATTRACTORS}

In a serially connected STNO system, the parameter region for synchronization is predicted not to be large. ${ }^{32}$ We therefore first investigate the synchronization of two identical STNOs. In the synchronization state, where the two STNOs have a common frequency $\tilde{\Omega}$ and a time lag $\delta$ (phase shift), the current given by Eq. (2) should have the same value upon interchanging the two STNOs: $\left.J(t)\right|_{\cos \psi_{2}(t)=\cos \psi_{1}(t+\delta)}=$ $\left.J(t)\right|_{\cos \psi_{2}(t)=\cos \psi_{1}(t-\delta)}$. Therefore, both in-phase $(\delta=0)$ and antiphase $(\delta=T / 2$, half the period) synchronization are possible. Our numerical simulations show that both are stable across a broad range of parameters, as shown in Fig. 2. This case is totally different from the typical coupled phase oscillators with the form $d \psi_{1,2} / d t=\Omega_{1,2}+K \sin \left(\psi_{2,1}-\psi_{1,2}\right)$. When $\Omega_{1}=$
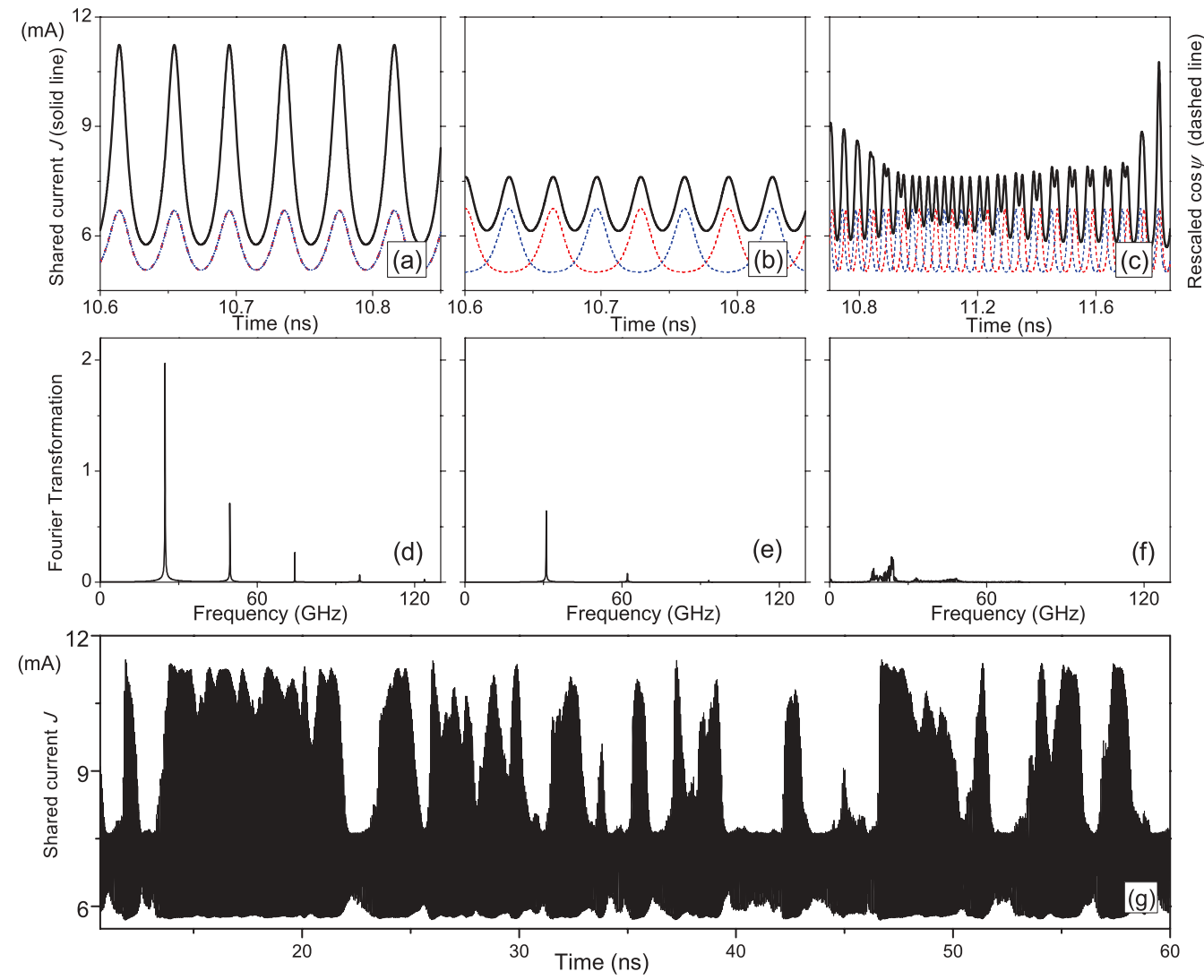

FIG. 2. (Color online) The coexistence of in-phase (a) and (d) and antiphase (b) and (e) synchronizations of two identical STNOs. In the first row, the solid lines are the shared currents $J$, and the dashed lines are the time serials of cos $\psi_{i}$ for the two STNOs. (d) and (e) The resonance peaks in the Fourier spectra of $J$ can represent the resonance peaks in the power spectra in experiments. When a small noise is introduced, the system randomly switches between these two attractors, inducing the resonance peak to vanish, as shown in (f). Panel (g) shows the time serials with noise. In order to give a clear illustration of the switching phenomenon, we show a fragment of $(\mathrm{g})$ in panel (c). 

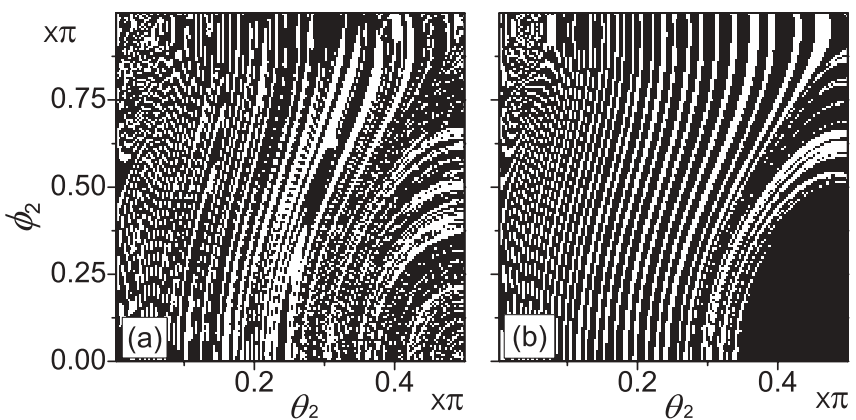

FIG. 3. (a) A section of the attracting basin of the antiphase (white) and in-phase (black) synchronization in the $\theta_{2}-\phi_{2}$ plane in a pair of serially connected STNO system. (b) A section of the attracting basin of the antiphase synchronization (white) and desynchronization (black). $\theta$ and $\phi$ are, respectively, the polar and azimuth angles of $\mathbf{m}$ in a spherical coordinate. Both the initial $\theta_{1}$ and $\phi_{1}$ are equal to $\pi / 2$. The system parameters of panels (a) and (b) are shown in Fig. 5, respectively.

$\Omega_{2}$, the attractor only depends on $K$. The mono-attractor will be either in-phase synchronization when $K>0$ or antiphase synchronization when $K<0 .{ }^{33}$ In Figs. 2(a) and 2(b), one can also see that the in-phase and antiphase synchronizations correspond to the stable $1: 1$ and $2: 1$ lockings of an STNO to the shared current $J(t)$, respectively. The fractional locking of an STNO to an external signal has also been reported in experiments, ${ }^{34,35}$ and cannot be described by means of coupled phase oscillators. $^{36}$

Because of the coexistence of these two attractors, the system may still exhibit different oscillations, despite being synchronized. From the Fourier spectra, one can observe that both the synchronized frequencies and the amplitudes are different. Through numerical simulation, we also found that the attracting basins of the two attractors are entangled in a complicated manner, as shown in Fig. 3(a). For two nonidentical STNOs, the entangled attracting basins are also present, with some changes in the details. Furthermore, across a wide region of parameters, the attractors can be antiphase even if the initial conditions of these two STNOs are identical. In experiments it is therefore not an easy task to control the initial conditions in order to favor either of the attractors. This may require some more advanced methods and sophisticated technologies.

The entangled attracting basins will have more influence on the experiments in the presence of noise perturbations, which are inevitable in experiments. In this case, noise can induce switching among these attractors, which is totally different from the mono-attractor case in which noise only induces a smearing of the attractor. In this STNO system, switching can be observed even with a very small noise, as shown in Figs. 2(c) and $2(\mathrm{~g})$. In this simulation, we simply add a Gaussian white noise term $\xi(t)$ to Eq. (1) to refer to various kinds of stochastic factors which occur in experiments, including, but not limited to, temperature. The standard deviation of $\xi(t)$ is quite smaller compared to the deterministic terms, for example, in Figs. 2(c), $2(\mathrm{f})$, and $2(\mathrm{~g})$, we use $\langle\xi(t) \xi(s)\rangle=2 \times 10^{-4} \times \frac{\gamma}{1+\alpha^{2}} \delta(t-s)$. Thus synchronization can be identified when measuring the average frequencies of the two STNOs. However, the resonance peak in the Fourier spectra nearly vanishes. This switching behavior does not have an intrinsic major frequency, as shown in Fig. 2(g). In such a nanosized high-frequency system, measurement of the resonance peak is commonly used to identify the synchronization state from the power spectra in experiments. The result in Fig. 2(f) means that this type of synchronization will require other identification methods. Moreover, although these nontrivial characteristics may have potential in some applications, if the original motivation of synchronizing is to increase the resonance power, this type of synchronization does not necessarily fulfil that goal.

When more than two STNOs are serially coupled, the situation becomes more complex. When an artificial time-delay $D$ is introduced in $J(t)=J_{d} /\left[C_{a}-C_{b} \sum_{i} \cos \psi_{i}(t-D)\right]$ in order to enlarge the synchronization region, ${ }^{21,32}$ further types of synchronization attractors will be observed. For example, when four STNOs are synchronized, we have observed one attractor in which all four STNOs completely synchronize with uniform frequency and zero phase difference, another attractor in which the four STNOs are divided into two antiphase groups, each of which consists of two in-phase STNOs, and even another in which three of the STNOs completely synchronize with uniform frequency $\tilde{\Omega}$, while the fourth one fractionally synchronizes with the others at $\frac{3}{4} \tilde{\Omega}$, and so on. The attracting basins are entangled in a higher-dimensional space, which may lead to further impact on experiments and applications.

\section{BIFURCATION TO SYNCHRONIZATION}

In the following, we analyze the phenomena of the coexisting attractors, from the viewpoint of dynamical system. Starting with the two coupled nonidentical STNOs, we investigate the bifurcation process, where synchronization is achieved by decreasing the difference between the two devices. We set a parameter mismatch $\Delta H_{d z}$ to characterize the difference, where $H_{d z_{1,2}}=H_{d z_{0}} \pm \Delta H_{d z} / 2$. If $\Delta H_{d z}$ decreases to zero, the case reduces to the identical STNOs pair. We have always found the coexistence of in-phase and antiphase synchronization attractors when the two STNOs are identical (i.e., $\Delta H_{d z}=0$ ). However, when $\Delta H_{d z} \neq 0$ for nonidentical STNOs pair, in-phase synchronization can emerge either before or after antiphase synchronization (i.e., the critical value of $\Delta H_{d z}$ for the onset of in-phase synchronization can be either bigger or smaller than that of antiphase synchronization, depending on other system parameters).

To study the bifurcation process, we construct a threedimensional Poincaré section: The azimuth angle of the first device $\phi_{1}=2 k \pi$, where $k$ is an integer. The desynchronization (or synchronization) state, which is a two-dimensional torus (or one-dimensional line) in this system, exhibits a onedimensional loop (or zero-dimensional point) on this section. To be clear, in the rest of this paper, if we just plot two of the total three dimensions of the Poincaré section, we preserve the two-dimensional desynchronization and one-dimensional synchronization states; otherwise, if we plot one dimension of the Poincaré section, we construct another cross section again and thus the desynchronization state will exhibit as two points and the synchronization state as one point.

As we mentioned, there are two cases in this system, depending on the system parameters. In the first case, as shown 

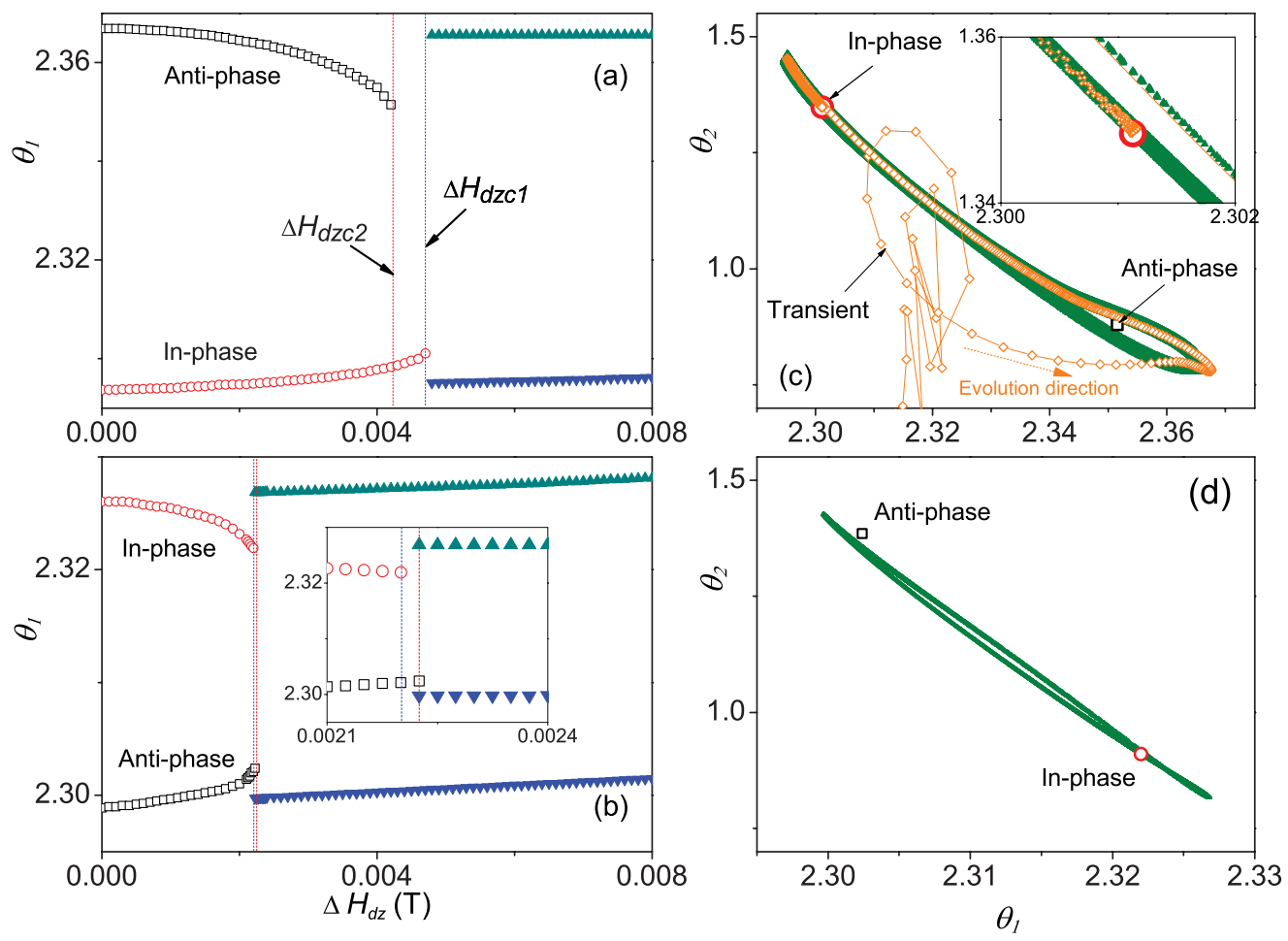

FIG. 4. (Color online) The two cases of bifurcation process: (a) and (c) the in-phase synchronization is achieved first when decreasing $\Delta H_{d z}$, and (b) and (d) the antiphase synchronization is achieved first, where (a) and (c) $\Delta J=3.75 \mathrm{~mA}$ and (b) and (d) $\Delta J=1.65 \mathrm{~mA}$, respectively. A Poincaré section is constructed as the azimuth angle of the first device $\phi_{1}=2 k \pi$. Its projection on the plane $\theta_{1}-\theta_{2}$ can be seen in panels (c) and (d), where the three attractors are of different system parameters: (c) $\Delta H_{d z}=0.0048 \mathrm{~T}$ (near the onset of the in-phase synchronization where the critical value $\Delta H_{d z c 1} \approx 0.0047 \mathrm{~T}$ ) for the desynchronization state (green limit cycle), $\Delta H_{d z}=0.0047 \mathrm{~T}$ for the in-phase state (red circle) and its transient process (orange diamonds connected by solid lines), and $\Delta H_{d z}=0.0042 \mathrm{~T}$ (near the onset of the antiphase synchronization) for the antiphase state (black square); (d) $\Delta H_{d z}=0.002225 \mathrm{~T}$ for the coexistence of desynchronization (green limit cycle) and antiphase synchronization (black square) and $\Delta H_{d z}=0.0022 \mathrm{~T}$ (near the onset of the in-phase synchronization) for the in-phase state (red circle). In panels (a) and (b), we demonstrate the bifurcation processes when decreasing $\Delta H_{d z}$. The equilateral and inverted triangles are the maximum and minimum values of $\theta_{1}$ of the limit cycle on the Poincaré section, and the red circles and black squares are the in-phase (red circle) and antiphase (black square) attractors, respectively. In the inset of panel (b), we enlarge the region around the bifurcation points, and in the inset of panel (c), we zoom in the region where the system evolves into the in-phase synchronization attractor.

in Fig. 4(a), when the parameter difference $\Delta H_{d z}$ is decreased, the desynchronization attractor immediately disappears accompanied by the emergence of a synchronization attractor at a critical parameter $\Delta H_{d z c 1}$. With further investigation, we know that this synchronization state is the in-phase one. However, with further decreasing $\Delta H_{d z c 1}$ to another critical parameter $\Delta H_{d z c 2}$, a new synchronization state (antiphase) can emerge. Meanwhile, the in-phase synchronization state still exists.

At the first bifurcation point $\Delta H_{d z c 1}$, the emergence of the in-phase synchronization is on the disappeared limit cycle, shown in Fig. 4(c), consistent with a saddle-node homoclinic bifurcation (a special case of saddle-node bifurcation, where a stable node-type solution and an unstable saddle-type solution emerge together on the limit cycle, so that the limit cycle turns into two heteroclinic orbits). In order to further check the bifurcation process, we plot a certain range of transient points in Fig. 4(c). It is clearly seen that the system evolves along a trajectory near the disappeared limit cycle towards the in-phase synchronization state, consistent with the birth of the heteroclinic orbits. These heteroclinic orbits and the saddles bring some complexity to the manifolds of the system, facilitating the complicated manner of the entangled attracting basins when the antiphase synchronization emerges, after the system reaches the second bifurcation point $\Delta H_{d z c 2}$. The attracting basin of the antiphase synchronization increases from zero to a certain portion of the whole phase space until $\Delta H_{d z}=0$. The onset of antiphase synchronization is therefore a typical saddle-node bifurcation.

On the other hand, there can be the second case, where the antiphase synchronization is achieved firstly when the parameter difference $\Delta H_{d z}$ is decreased, as shown in Fig. 4(b). However, the desynchronization attractor still exists, such that we can get the coexistence of the antiphase synchronization and desynchronization in some regions of system parameters, as shown in Fig. 4(d). This phenomenon is quite similar to the case of synchronization in the injected STNO system. ${ }^{37-39}$ One example of the attracting basins of this type of coexisting attractors is shown in Fig. 3(b), which also exhibits the similar pattern to that of the injected STNO system [compared to Fig. 8(C) in Ref. 39]. In one part of the phase space [the lower right corner in Fig. 3(b)], the system is always attracted to the desynchronization attractor, whereas in the remaining part the attracted basins are entangled together. The reason is that the desynchronization orbit is a two-dimensional tori in the 


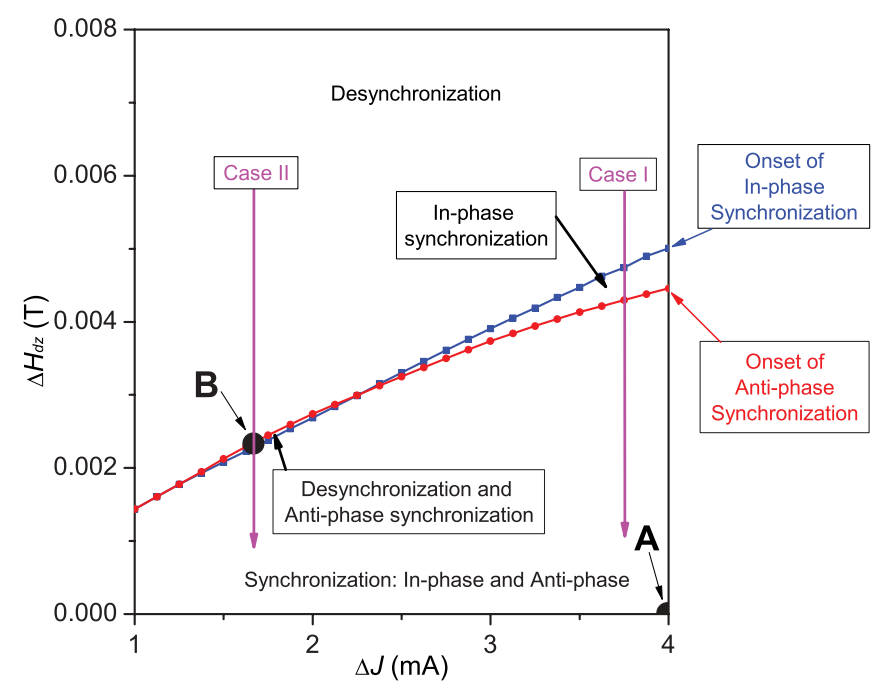

FIG. 5. (Color online) The bifurcation process in achieving synchronization for different shared currents. [Case I (correspondingly II)]The onset of in-phase synchronization occurs before (or after) that of antiphase synchronization when the difference between the two STNO devices is reduced. $\Delta J$ denotes the amplitude of the shared currents, which stands for the coupling strength between the two STNOs. The two points $\mathbf{A}$ and $\mathbf{B}$ show the two parameter points for the states, respectively, as shown in Figs. 3(a) and 3(b).

phase space which separates the whole phase space into two parts. When the antiphase synchronization is achieved via a saddle-node bifurcation, its attracting basin cannot spread through the tori. In a general case, the fate of the tori usually develops in one of two ways. In the first situation, it disappears via a homoclinic bifurcation. It should be noted that this type of bifurcation does not exist for our system of a chain of electrically coupled STNOs. However, synchronization in the injected STNO system is an example of this case. In the second situation, the tori disappears itself via a bifurcation process, resulting in the stabilizing of the other synchronization attractor. The attracting basins of the two synchronization attractors are entangled throughout nearly the whole space. We find that the serially connected STNO system studied in this work always belongs to this situation (i.e., when the parameter difference $\Delta H_{d z}$ is further decreased, inphase synchronization is achieved via saddle-node homoclinic bifurcation), as shown in Figs. 4(b) and 4(d).

Actually, for both cases, the onsets of these two types of bifurcation processes do not show dependence on each other. But it should be noted that both of them will happen when $\Delta H_{d z}$ decreases, so that we always find the coexistence of the two synchronization attractors when two identical STNOs are serially connected. The whole picture of the bifurcation processes is shown in Fig. 5, where the two aforementioned cases can be easily found.

To sum up, the synchronization of serially connected STNOs system is achieved via a saddle-node bifurcation. A type of characteristic of the saddle-node bifurcation is that one synchronization attractor emerges together with another unstable orbit, rather than the desynchronization or other synchronization attractors necessarily disappearing, so that the coexistence of multiple synchronization attractors is a common phenomenon. ${ }^{37,38,40-44}$ Furthermore, the manifolds in the phase space may be very complicated if heteroclinic or homoclinic orbits emerge together with the saddle-node bifurcation. As is shown in this work, the attracting basins are entangled together, which have serious impacts on experiments and applications of the system.

\section{CONCLUSIONS}

In a serially connected STNO system, we find that synchronization takes place via saddle-node bifurcation across quite a wide range of system parameters, resulting in the coexistence of multiple synchronization attractors. These coexistent attractors show different oscillatory frequencies and amplitudes. A very small noise can make the resonance peak vanish. These results have great significance for experiments and applications. For example, they pose a challenge to the common method of experimentally identifying synchronization in such nanosized high-frequency systems by measuring the resonance peak. Thus new methods to identify synchronization will be required; meanwhile, this type of synchronization does not necessarily increase the resonance peak, which was one of the original motivations for synchronizing STNOs. These effects on experiments and applications should be taken into serious consideration in similar nanosized high-frequency systems.

\section{ACKNOWLEDGMENTS}

This work is supported by Hong Kong Baptist University and conducted using the resources of the High Performance Cluster Computing Centre, Hong Kong Baptist University, which receives funding from Research Grant Council, University Grant Committee of the HKSAR, and HongKong Baptist University. J.A. acknowledges support from the Swedish Research Council, the Swedish Foundation for Strategic Research (Future Research Leader Programme), and the Göran Gustafsson Foundation. J.A. is a Royal Swedish Academy of Sciences Research Fellow supported by a grant from the Knut and Alice Wallenberg Foundation. *cszhou@hkbu.edu.hk

${ }^{1}$ J. A. Katine, F. J. Albert, R. A. Buhrman, E. B. Myers, and D. C. Ralph, Phys. Rev. Lett. 84, 3149 (2000).

${ }^{2}$ D. C. Ralph and M. D. Stiles, J. Magn. Magn. Mater. 321, 2508 (2009).
${ }^{3}$ M. R. Pufall, W. H. Rippard, S. Kaka, T. J. Silva, and S. E. Russek, Appl. Phys. Lett. 86, 082506 (2005).

${ }^{4}$ T. J. Silva and W. H. Rippard, J. Magn. Magn. Mater. 320, 1260 (2008).

${ }^{5}$ Y. Zhou, J. Persson, S. Bonetti, and J. Åkerman, Appl. Phys. Lett. 92, 092505 (2008). 
${ }^{6}$ Y. Zhou and J. Åkerman, Appl. Phys. Lett. 94, 112503 (2009).

${ }^{7}$ R. B. Karabalin, M. C. Cross, and M. L. Roukes, Phys. Rev. B 79, 165309 (2009).

${ }^{8}$ M. L. Roukes, Phys. World 14, 25 (2001).

${ }^{9}$ D. Rugar, R. Budakian, H. J. Mamin, and B. W. Chui, Nature (London) 430, 329 (2004).

${ }^{10}$ A. N. Cleland and M. L. Roukes, Nature (London) 392, 160 (1998).

${ }^{11}$ M. Madami, S. Bonetti, G. Consolo, S. Tacchi, G. Carlotti, G. Gubbiotti, F. B. Mancoff, M. A. Yar, and J. Åkerman, Nat. Nanotechnol. 6, 635 (2011).

${ }^{12}$ Y. Pogoryelov, P. K. Muduli, S. Bonetti, E. Iacocca, F. Mancoff, and J. Åkerman, Appl. Phys. Lett. 98, 192501 (2011).

${ }^{13}$ M. P. Kostylev, A. A. Serga, T. Schneider, B. Leven, and B. Hillebrands, Appl. Phys. Lett. 87, 153501 (2005).

${ }^{14}$ K. S. Lee and S. K. Kim, J. Appl. Phys. 104, 053909 (2008).

${ }^{15}$ T. Schneider, A. A. Serga, B. Leven, B. Hillebrands, R. L. Stamps, and M. P. Kostylev, Appl. Phys. Lett. 92, 022505 (2008).

${ }^{16}$ A. Khitun, M. Q. Bao, and K. L. Wang, J. Phys. D: Appl. Phys. 43, 264005 (2010).

${ }^{17}$ A. Khitun, M. Q. Bao, and K. L. Wang, IEEE Trans. Magn. 44, 2141 (2008).

${ }^{18}$ F. Macia, A. D. Kent, and F. C. Hoppensteadt, Nanotechnology 22, 095301 (2011).

${ }^{19}$ A. Ruotolo, V. Cros, B. Georges, A. Dussaux, J. Grollier, C. Deranlot, R. Guillemet, K. Bouzehouane, S. Fusil, and A. Fert, Nat. Nanotechnol. 4, 528 (2009).

${ }^{20}$ J. Grollier, V. Cros, and A. Fert, Phys. Rev. B 73, 060409(R) (2006).

${ }^{21}$ J. Persson, Y. Zhou, and J. Åkerman, J. Appl. Phys. 101, 09A503 (2007).

${ }^{22}$ A. Slavin, Nat. Nanotechnol. 4, 479 (2009).

${ }^{23}$ Y. Zhou, V. Tiberkevich, G. Consolo, E. Iacocca, B. Azzerboni, A. Slavin, and J. Åkerman, Phys. Rev. B 82, 012408 (2010).

${ }^{24}$ S. Kaka, M. R. Pufall, W. H. Rippard, T. J. Silva, S. E. Russek, and J. A. Katine, Nature (London) 437, 389 (2005).
${ }^{25}$ F. B. Mancoff, N. D. Rizzo, B. N. Engel, and S. Tehrani, Nature (London) 437, 393 (2005).

${ }^{26}$ S. M. Rezende, F. M. de Aguiar, R. L. Rodríguez-Suárez, and A. Azevedo, Phys. Rev. Lett. 98, 087202 (2007).

${ }^{27}$ W. H. Rippard, M. R. Pufall, S. Kaka, T. J. Silva, S. E. Russek, and J. A. Katine, Phys. Rev. Lett. 95, 067203 (2005).

${ }^{28}$ B. Georges, J. Grollier, M. Darques, V. Cros, C. Deranlot, B. Marcilhac, G. Faini, and A. Fert, Phys. Rev. Lett. 101, 017201 (2008).

${ }^{29}$ J. C. Slonczewski, J. Magn. Magn. Mater. 159, L1 (1996).

${ }^{30}$ S. Kiselev, J. Sankey, I. Krivorotov, N. Emley, R. Schoelkopf, R. Buhrman, and D. Ralph, Nature (London) 425, 380 (2003).

${ }^{31}$ J. Z. Sun, Phys. Rev. B 62, 570 (2000).

${ }^{32}$ D. Li, Y. Zhou, C. Zhou, and B. Hu, Phys. Rev. B 82, 140407(R) (2010).

${ }^{33}$ H. G. Schuster and P. Wagner, Prog. Theor. Phys. 81, 939 (1989).

${ }^{34}$ S. Urazhdin, P. Tabor, V. Tiberkevich, and A. Slavin, Phys. Rev. Lett. 105, 104101 (2010).

${ }^{35}$ A. Dussaux, A. V. Khvalkovskiy, J. Grollier, V. Cros, A. Fukushima, M. Konoto, H. Kubota, K. Yakushiji, S. Yuasa, K. Ando et al., Appl. Phys. Lett. 98, 132506 (2011).

${ }^{36}$ D. Li, Y. Zhou, C. Zhou, and B. Hu, Phys. Rev. B 83, 174424 (2011).

${ }^{37}$ C. Sperpico, R. Bonin, G. Bertotti, M. d'Aquino, and I. D. Mayergoyz, IEEE Trans. Magn. 45, 3441 (2009).

${ }^{38}$ M. d'Aquino, C. Serpico, R. Bonin, G. Bertotti, and I. D. Mayergoyz, Phys. Rev. B 82, 064415 (2010).

${ }^{39}$ D. Li, Y. Zhou, B. Hu, and C. Zhou, Phys. Rev. B 84, 104414 (2011).

${ }^{40}$ A. Pikovsky, G. Osipov, M. Rosenblum, M. Zaks, and J. Kurths, Phys. Rev. Lett. 79, 47 (1997).

${ }^{41}$ Z. Zheng, G. Hu, and B. Hu, Phys. Rev. Lett. 81, 5318 (1998).

${ }^{42}$ A. Pikovsky, M. Rosenblum, and J. Kurths, Int. J. Bifurcation Chaos 10, 2291 (2000).

${ }^{43}$ Y. Maistrenko, O. Popovych, O. Burylko, and P. A. Tass, Phys. Rev. Lett. 93, 084102 (2004)

${ }^{44}$ R. Tönjes and B. Blasius, Phys. Rev. E 80, 026202 (2009). 